\title{
Is de Sitter space always excluded in semiclassical $f(R)$ gravity?
}

\author{
Roberto Casadio $^{a b *}$, Andrea Giugno ${ }^{c \dagger}$, Andrea Giusti ${ }^{d \ddagger}$ and Valerio Faraoni ${ }^{d \S}$ \\ ${ }^{a}$ Dipartimento di Fisica e Astronomia, Università di Bologna \\ via Irnerio 46, I-40126 Bologna, Italy \\ ${ }^{b}$ I.N.F.N., Sezione di Bologna, I.S. FLAG \\ via B. Pichat 6/2, I-40127 Bologna, Italy \\ ${ }^{c}$ Arnold Sommerfeld Center, Ludwig-Maximilians-Universität \\ Theresienstraße 3\%, 80333 München, Germany \\ ${ }^{d}$ Department of Physics and Astronomy, Bishop's University, \\ 2600 College Street, Sherbrooke Québec, Canada J1M 1Z7
}

19th June 2019

\begin{abstract}
It is shown that the recent corpuscular description of gravity generically excludes de Sitter spacetime in any semiclassical version of $f(R)$ gravity. A phantom phenomenology of the cosmic dynamics is also naturally excluded.
\end{abstract}

\footnotetext{
*E-mail: casadio@bo.infn.it

$\dagger$ E-mail: A.Giugno@physik.uni-muenchen.de

$\ddagger$ E-mail: agiusti@ubishops.ca

§E-mail: vfaraoni@ubishops.ca
} 


\section{Introduction}

The inflationary scenario, whose original formulation was initially advocated by Starobinsky [1,2] and Guth [3, finds its fundamental motivation in the quest for the resolution of three major conceptual issues in early universe cosmology, namely the homogeneity and flatness of our universe, and the magnetic-monopole problem. Later on, Linde 4, Albrecht, and Steinhardt [5 built on this proposal introducing a scheme in which the accelerated expansion was driven by a scalar field, commonly referred to as the inflaton, rolling slowly on a plateau of the potential toward its minimum. Scalar field inflation has the added value of providing a mechanism for the generation of density perturbations that constitute the seeds for structures that grow in the later dust-dominated era. The key idea behind scalar field inflation is that, if this plateau is sufficiently flat, the phase of (quasi-)exponential expansion lasts long enough to solve the cosmological problems mentioned above, providing also a mechanism for the universe to gracefully leave this highly accelerated phase. This last paradigm can be recast as a $f(R)$ theory of gravity (see, e.g., [6] 9]) and it has become part of the standard picture of the early universe. The Starobinsky model [1,2] appears to be particularly favoured by present observations [10,11].

In inflationary theory, it is common practice to employ the semiclassical picture of gravity, which restricts the picture to the dynamics of quantum fields on a classical curved spacetime. Further, only quantum fluctuations of the fields around classical values are allowed. However, this approximation inevitably fails to capture relevant quantum properties of gravity in the very early universe [12. A way to preserve a quantum mechanical description of the early universe consists of conceiving the classical spacetime geometry as an emergent, rather than a fundamental, property of nature. A scheme that successfully implements this picture at both astrophysical and cosmological scales is the so-called corpuscular theory of gravity [13 16]. Its fundamental idea is that a classical spacetime can be thought of as a self-sustained marginally bound state of a large number of soft off-shell gravitons. The high multiplicity of this state allows one to recover the classical and semiclassical features of the emergent spacetime. The first implementation of the corpuscular model [13 16] investigated the physics hidden inside a black hole's event horizon. Precisely, within this framework a black hole of mass $M$ is understood as a marginally bound state of $N$ off-shell gravitons of typical Compton length $\lambda_{\mathrm{G}}$ of the order of the Schwarzschild radius $R_{\mathrm{H}}=2 G_{\mathrm{N}} M$. 11 Then, even when the system enters the strong coupling regime, the requirement of a marginally bound state allows us to frame this theoretical set-up as a Newtonian theory of many gravitons that appear to be loosely confined in a potential well of size $\lambda_{\mathrm{G}} \simeq R_{\mathrm{H}}$. As a consequence, the effective gravitational coupling among the constituent gravitons of this bound state scales like $\alpha \sim 1 / N$. This result is of paramount importance for this whole picture since it allows one to understand classical black holes as the result of bound states of gravitons on the verge of a quantum phase transition. Indeed, while the effective coupling $\alpha$ is very small, the collective coupling for the system $g=N \alpha \sim 1$, and this configuration clearly matches the traditional picture of an interacting Bose-Einstein condensate at the critical point. This ultimately allows us to provide a corpuscular interpretation of Hawking radiation in terms of gravitons leaking out of the bound state [13 16]. Further, working in this general framework, it is possible to recover the correct post-Newtonian expansion of the gravitational field generated by a static, spherically symmetric source [17,18] in a fully quantum framework and the Bekenstein-Hawking area law [19, including semiclassical logarithmic corrections [20, 21] for the Hawking radiation. What is more, when applied to the case of maximally symmetric spaces, the corpuscular theory of gravity has proven effective in reproducing the main features of inflation [12,22, and dark matter phenomenology [23,24]. In particular, it was shown in Refs. [25,26] that Starobinsky's inflation is naturally embedded in this framework.

In this work, we show that a corpuscular description of $f(R)$ theories of gravity always excludes the exact de Sitter spacetime, thus generalizing the results obtained in Ref. [27, while providing some (fully quantum mechanical) insight on the physically admissible $f(R)$ models.

\footnotetext{
${ }^{1}$ We shall use units with $c=1$ and the Newton constant $G_{\mathrm{N}}=\ell_{\mathrm{p}} / m_{\mathrm{p}}$, where $\ell_{\mathrm{p}}$ and $m_{\mathrm{p}}$ are the Planck length and mass, respectively, and the reduced Planck constant is $\hbar=\ell_{\mathrm{p}} m_{\mathrm{p}}$.
} 


\section{2 de Sitter universe}

It is well known that the de Sitter metric is an exact solution of the modified theory of gravity [7, 8,28$]$

$$
S=\frac{1}{16 \pi G_{\mathrm{N}}} \int \mathrm{d}^{4} x \sqrt{-g} f(R),
$$

with $[29,30$,

$$
f(R)=\gamma \ell_{\mathrm{p}}^{2} R^{2},
$$

where $\gamma$ is a dimensionless constant. We recall that the equation of motion arising from the variation of the action (11) for a spatially flat Friedmann-Lemaître-Robertson-Walker (FLRW) metric,

$$
\mathrm{d} s^{2}=-\mathrm{d} t^{2}+a^{2}(t)\left(\mathrm{d} r^{2}+r^{2} \mathrm{~d} \Omega^{2}\right),
$$

is 6 6, $9,29,31$ ]

$$
6 f^{\prime}(R) H^{2}=R f^{\prime}(R)-f(R)-6 H \dot{R} f^{\prime \prime}(R),
$$

where a prime and an overdot denote differentiation with respect to $R$ and to the comoving time $t$, respectively. In particular, for the theory (2), one obtains

$$
12 R H^{2}=R^{2}-12 H \dot{R}
$$

and, for de Sitter spacetime with $a(t)=\mathrm{e}^{\sqrt{\Lambda / 3} t}$ and constant $H \equiv \dot{a} / a=\sqrt{\Lambda / 3}$, one has

$$
\dot{R}=24 H \dot{H}=0 \text {. }
$$

and

$$
\frac{R}{12}=\frac{\dot{H}+2 H^{2}}{2}=H^{2}=\frac{\Lambda}{3} .
$$

Integrating the left hand side of Eq. (7) over a sphere of Hubble radius $L_{\Lambda}=H_{\Lambda}^{-1}=\sqrt{3 / \Lambda}$ yields

$$
L_{\Lambda}^{3} H_{\Lambda}^{2} \simeq L_{\Lambda} \equiv-G_{\mathrm{N}} U_{\mathrm{N}}
$$

and, likewise, the right hand side of Eq. (77) yields

$$
L_{\Lambda}^{3}(\Lambda / 3) \simeq L_{\Lambda} \equiv G_{\mathrm{N}} U_{\mathrm{PN}},
$$

where we introduced a "Newtonian" and a "post-Newtonian" (or post-Minkowskian) energy $U_{\mathrm{N}}$ and $U_{\mathrm{PN}}$, respectively 2 These expressions will be our starting point to investigate the relation between corpuscular model and de Sitter space.

\section{Corpuscular de Sitter and depletion}

Let us begin by assuming that matter and the corpuscular state of gravitons together reproduce the Friedmann equation of cosmology, which we write as the Hamiltonian constraint

$$
\mathcal{H}_{\mathrm{M}}+\mathcal{H}_{\mathrm{G}}=0
$$

\footnotetext{
${ }^{2}$ Factors of order unity will be often omitted from now on.
} 
where $\mathcal{H}_{\mathrm{M}}$ is the matter energy and $\mathcal{H}_{\mathrm{G}}$ is the analogue quantity for the graviton state. Local (Newton or Einstein) gravity being attractive implies that $\mathcal{H}_{\mathrm{G}} \leq 0$, although this is not true for the graviton selfinteraction [17, 18], and might not be true for the cosmological condensate of gravitons as a whole, as we are now going to discuss.

In order to obtain the de Sitter spacetime in general relativity, that is the theory (11) with $f(R)=R$, one must add a cosmological constant term, or vacuum energy density $\rho_{\Lambda}$, so that the Friedmann equation,

$$
3 H^{2}=8 \pi G_{\mathrm{N}} \rho_{\Lambda},
$$

equals precisely Eq. (77). Upon integrating again on the volume inside the Hubble radius, one has

$$
L_{\Lambda} \simeq G_{\mathrm{N}} L_{\Lambda}^{3} \rho_{\Lambda} \simeq \ell_{\mathrm{p}} \frac{M_{\Lambda}}{m_{\mathrm{p}}},
$$

which looks exactly like the expression of the horizon radius for a black hole of mass $M_{\Lambda}$, and is the reason why it was conjectured that de Sitter spacetime could likewise be viewed as a graviton condensate [12].

One can roughly describe the corpuscular model by assuming that the (soft, virtual) graviton selfinteraction gives rise to a condensate of $N_{\Lambda}$ gravitons of typical Compton length $\lambda \simeq L_{\Lambda}$, so that $M_{\Lambda}=$ $N_{\Lambda} \ell_{\mathrm{p}} m_{\mathrm{p}} / L_{\Lambda}$, and the usual consistency condition

$$
M_{\Lambda} \sim \sqrt{N_{\Lambda}} m_{\mathrm{p}}
$$

for the graviton condensate immediately follows from Eq. (12). Equivalently, one finds

$$
L_{\Lambda} \sim \sqrt{N_{\Lambda}} \ell_{\mathrm{p}},
$$

which shows that for a macroscopic universe one needs $N_{\Lambda} \gg 1$. The above relations do not need to hold for gravitons that do not belong to the condensate, therefore one expects deviations to occur if regular matter is added [23,24], or if the system is driven out of equilibrium.

We can refine the above corpuscular description of de Sitter by following the line of reasoning of Refs. 17, 18, where it was shown that the maximal packing condition yielding the scaling relation (14) for a black hole actually follows from the energy balance (10) when matter becomes totally negligible. In the present case, matter is absent a priori and $\mathcal{H}_{\mathrm{M}}=0$, so that one is left with

$$
\mathcal{H}_{\mathrm{G}} \simeq U_{\mathrm{N}}+U_{\mathrm{PN}}=0 .
$$

The negative "Newtonian energy" of the $N_{\Lambda}$ gravitons can be obtained from a coherent state description of the condensate [17,18] in which each graviton has negative binding energy $\varepsilon_{\Lambda}$ given by the Compton relation, that is

$$
U_{\mathrm{N}} \simeq M_{\Lambda} \phi_{\mathrm{N}}=N_{\Lambda} \varepsilon_{\Lambda}=-N_{\Lambda} \frac{\ell_{\mathrm{p}} m_{\mathrm{p}}}{L_{\Lambda}} .
$$

The positive "post-Newtonian" contribution is then given by the (bootstrapped) graviton self-interaction term [17,18,

$$
U_{\mathrm{PN}} \simeq N_{\Lambda} \varepsilon_{\Lambda} \phi_{\mathrm{N}}=N_{\Lambda}^{3 / 2} \frac{\ell_{\mathrm{p}}^{2} m_{\mathrm{p}}}{L_{\Lambda}^{2}},
$$

where we used the Newtonian potential

$$
\phi_{\mathrm{N}}=-\frac{N_{\Lambda} \ell_{\mathrm{p}} m_{\mathrm{p}}}{M_{\Lambda} L_{\Lambda}}=-\sqrt{N_{\Lambda}} \frac{\ell_{\mathrm{p}}}{L_{\Lambda}},
$$

as follows from Eq. (16) and the scaling relation (13). 
In an ideal de Sitter universe, gravitons should satisfy the balance condition (15). Let us rewrite the Hamiltonian (15) as

$$
\mathcal{H}_{\mathrm{G}}^{(2)} \simeq \beta\left(U_{\mathrm{N}}+U_{\mathrm{PN}}\right)
$$

corresponding to the effective metric action (11) with Eq. (2). Here we have introduced the dimensionless parameter $\beta>0$ of order unity in order to keep track of this contribution. Let us include a term corresponding to the Einstein-Hilbert action, that is

$$
\mathcal{H}_{\mathrm{G}}^{(1)} \simeq \alpha U_{\mathrm{N}},
$$

where $\alpha>0$. The full energy balance is therefore

$$
\mathcal{H}_{\mathrm{G}}=\mathcal{H}_{\mathrm{G}}^{(1)}+\mathcal{H}_{\mathrm{G}}^{(2)} \simeq(\alpha+\beta) U_{\mathrm{N}}+\beta U_{\mathrm{PN}}=0
$$

and, because of the term proportional to $\alpha$, we expect the expressions (8) and (9) for the ideal de Sitter condensate to no longer constitute a solution. In fact, we are interested in a stage when departures from the de Sitter scalings are small, and we can therefore assume that the potentials now take the slightly more general form

$$
G_{\mathrm{N}} U_{\mathrm{N}} \simeq-L^{3} H^{2}
$$

and

$$
G_{\mathrm{N}} U_{\mathrm{PN}} \simeq L^{3} L_{\Lambda}^{-2}
$$

where $L \sim L_{\Lambda}$ is the new Hubble radius. Substitution into Eq. (21) yields

$$
L^{3}\left[-(\alpha+\beta) H^{2}+\beta L_{\Lambda}^{-2}\right] \simeq 0
$$

which is solved by

$$
H^{2} \simeq \frac{\beta}{\alpha+\beta} \frac{1}{L_{\Lambda}^{2}}
$$

Of course, the de Sitter case is properly recovered when $\alpha=0$, but $\alpha>0$ implies that $H<H_{\Lambda}$ as expected. If the system starts with $H \simeq H_{\Lambda}$, the time derivative $\dot{H}$ must be negative (i.e., the universe does not superaccelerate) in order to ensure the constraint (21) holds at all times. This can be seen explicitly by writing

$$
H=H_{\Lambda}+\dot{H} \delta t
$$

where the typical time scale $\delta t \simeq L_{\Lambda}$, since gravitons of Compton length $L_{\Lambda}$ cannot be sensitive to shorter times. Equation (24) finally yields

$$
\dot{H} \simeq-\frac{\alpha}{\alpha+\beta} \frac{H_{\Lambda}}{\delta t} \simeq-\frac{\alpha}{\alpha+\beta} \frac{1}{L_{\Lambda}^{2}} .
$$

Further, the slow-roll parameter in the corpuscular model is

$$
\epsilon \equiv-\frac{\dot{H}}{H^{2}} \simeq \frac{\alpha}{\alpha+\beta}
$$

and one obtains $\epsilon=0$ in the limit $\alpha \rightarrow 0$, provided the quantum depletion can be neglected.

In detail, gravitons in the condensate generate the effective Hubble parameter $H \sim N_{\Lambda}^{-1 / 2} \sim L_{\Lambda}^{-1}$, but they also scatter and deplete. Their number therefore changes in time, according to [12,22]

$$
\frac{\ell_{\mathrm{p}} \dot{N}_{\Lambda}}{\sqrt{N_{\Lambda}}}=\left.\frac{\ell_{\mathrm{p}} \dot{N}_{\Lambda}}{\sqrt{N_{\Lambda}}}\right|_{\text {eom }}+\left.\frac{\ell_{\mathrm{p}} \dot{N}_{\Lambda}}{\sqrt{N_{\Lambda}}}\right|_{\mathrm{q}},
$$


where the classical equation of motion gives (for $\alpha \neq 0$ )

$$
\left.\frac{\ell_{\mathrm{p}} \dot{N}_{\Lambda}}{\sqrt{N_{\Lambda}}}\right|_{\mathrm{eom}} \simeq-\frac{\dot{H}}{H^{2}} \simeq \frac{\alpha}{\alpha+\beta},
$$

and the purely quantum depletion yields

$$
-\frac{\dot{H}}{H^{2}}=\ell_{\mathrm{p}} \dot{M}_{\Lambda} \simeq-\frac{m_{\mathrm{p}}^{2}}{M_{\Lambda}^{2}} \simeq-\ell_{\mathrm{p}}^{2} H^{2} \simeq-\frac{\beta}{\alpha+\beta} \frac{1}{N_{\Lambda}} .
$$

Putting the two terms together we obtain, for $\alpha \ll \beta \simeq 1$,

$$
-\frac{\dot{H}}{H^{2}} \simeq \frac{\ell_{\mathrm{p}} \dot{N}_{\Lambda}}{\sqrt{N_{\Lambda}}} \simeq \alpha\left(1-\frac{\beta}{\alpha N_{\Lambda}}\right),
$$

leading to a critical value of $\alpha$

$$
\alpha \gtrsim \alpha_{\mathrm{c}} \simeq \frac{\beta}{N_{\Lambda}} \sim \frac{\ell_{\mathrm{p}}^{2}}{L_{\Lambda}^{2}},
$$

which can be interpreted as a minimum "distance" from de Sitter space.

Now, the Hamiltonian $\mathcal{H}_{G}$ for a polynomial $f(R)$ theory of gravity always consists of two contributions $\mathcal{H}_{G}^{(1)}$ and $\mathcal{H}_{G}^{(2)}$ in corpuscular gravity. Effectively, the universe moves away from a de Sitter fixed point of Eq. (21), $f(R) \simeq R^{2}$, and approaches asymptotically another fixed point of general relativity with positive cosmological constant, that is $f(R) \simeq R-\Lambda$ [23]25]. More precisely, assuming that $R \simeq \Lambda$ is approximately constant, Eq. (4) yields

$$
6 f^{\prime}(R) H^{2} \simeq R f^{\prime}(R)-f(R) .
$$

If we write

$$
f(R)=\sum_{k=1}^{N} a_{k} \ell_{\mathrm{p}}^{2 k-2} R^{k},
$$

we find

$$
R f^{\prime}(R)-f(R)=\sum_{k=2}^{N}(k-1) a_{k} \ell_{\mathrm{p}}^{2 k-2} R^{k}
$$

and, up to coefficients of order unity, Eq. (34) reduces to

$$
H^{2}\left(a_{1}+\sum_{k=2}^{N} k a_{k} \ell_{\mathrm{p}}^{2 k-2} R^{k-1}\right) \simeq \sum_{k=2}^{N}(k-1) a_{k} \ell_{\mathrm{p}}^{2 k-2} R^{k},
$$

where we singled out the coefficient $a_{1}$ for later convenience. Using $R \simeq \Lambda$ and defining $n=k-1$ yield

$$
\begin{aligned}
a_{1} H^{2} & \simeq \sum_{n=1}^{N-1}\left[n\left(\Lambda-H^{2}\right)-H^{2}\right] a_{n+1} \ell_{\mathrm{p}}^{2 n} \Lambda^{n} \\
& \simeq \sum_{n=1}^{N-1}\left(\Lambda-H^{2}\right) n a_{n+1} \ell_{\mathrm{p}}^{2 n} \Lambda^{n} .
\end{aligned}
$$

If we then use Eqs. (8) and (9), it is easy to see that

$$
H^{2} \simeq-\frac{G_{\mathrm{N}} U_{\mathrm{N}}}{L_{\Lambda}^{3}} \simeq-\frac{\ell_{\mathrm{p}}}{m_{\mathrm{p}} L_{\Lambda}^{3}} U_{\mathrm{N}}
$$


and

$$
\Lambda \simeq \frac{G_{\mathrm{N}} U_{\mathrm{PN}}}{L_{\Lambda}^{3}} \simeq \frac{\ell_{\mathrm{p}}}{m_{\mathrm{p}} L_{\Lambda}^{3}} U_{\mathrm{PN}},
$$

which lead to

$$
a_{1} U_{\mathrm{N}}+\left(U_{\mathrm{N}}+U_{\mathrm{PN}}\right) \sum_{n=1}^{N-1} n a_{n+1}\left(\frac{\ell_{\mathrm{p}}^{3} U_{\mathrm{PN}}}{L_{\Lambda}^{3} m_{\mathrm{p}}}\right)^{n} \simeq 0 .
$$

For the Starobinsky model $(N=2)$, the latter reads simply

$$
a_{1} U_{\mathrm{N}}+a_{2}\left(\frac{\ell_{\mathrm{p}}^{3} U_{\mathrm{PN}}}{L_{\Lambda}^{3} m_{\mathrm{p}}}\right)\left(U_{\mathrm{N}}+U_{\mathrm{PN}}\right) \simeq 0 .
$$

Now, recalling that $a_{1}=\alpha$ and $a_{2}=\gamma$, it is

$$
\alpha U_{\mathrm{N}}+\gamma \frac{\ell_{\mathrm{p}}^{2}}{L_{\Lambda}^{2}}\left(U_{\mathrm{N}}+U_{\mathrm{PN}}\right) \simeq 0,
$$

in which we further used the de Sitter value (9) for $U_{\mathrm{PN}}$, since we are interested in studying the stability of this space. Comparing with Eq. (21), one recovers

$$
\beta=\beta_{2} \equiv \gamma \frac{\ell_{\mathrm{p}}^{2}}{L_{\Lambda}^{2}} .
$$

Likewise, for the general Eq. (35), one obtains Eq. (21) with $\alpha=a_{1}$ and

$$
\beta=\beta_{N} \equiv \sum_{n=1}^{N-1} n a_{n+1}\left(\frac{\ell_{\mathrm{p}}}{L_{\Lambda}}\right)^{2 n} .
$$

All of the above estimates can be improved but, comparing with the previous discussion, we expect again a bound similar to the one in Eq. (33).

\section{Conclusions}

When the corpuscular model is studied in conjunction with quadratic corrections to the Einstein-Hilbert action, as is natural, and is applied to early universe cosmology (as in Starobinsky inflation, currently favored by cosmological observations), one finds that an exact de Sitter space is always excluded. This result is significant because de Sitter spacetime is the most common attractor in the FLRW cosmology based on general relativity and on $f(R)$ theories of gravity and, in order to be a fixed point, de Sitter space must be an exact solution of the cosmic dynamics.

From an effective point of view, in the corpuscular model a universe that is close to (but not exactly) de Sitter in the high curvature regime where $f(R) \propto R^{2}$ moves away from it due to gravitons leaking out of the bound state and approaches a different de Sitter space in the low curvature regime where $f(R) \simeq R-\Lambda$. If the universe was described at all times by a purely classical $f(R)$ theory, this beheavior would be described by a heteroclinic trajectory linking two de Sitter fixed points in phase space, but this is not the case in the corpuscular model with graviton depletion, in which the universe departs from the initial, high curvature, coherent state.

From a classical perspective, an $f(R)$ theory can be conformally mapped into Einstein gravity minimally coupled to a scalar field with a complicated potential, the latter is known as the Einstein conformal frame whereas the former defines the Jordan frame for the model. A key result of Ref. [12] is that the exclusion of the de Sitter space is a direct consequence of the quantum depletion in the Einstein frame. Here, instead, 
we offer a different angle to this result based on the Hamiltonian constraint of cosmology and the Jordan frame description of quantum depletion.

Finally, the cosmological phenomenology of the corpuscolar model is quite reasonable and not exotic, in the sense that phantom behavior is always avoided since $\dot{H}$ must be negative and, in addition to never reaching a de Sitter state, the universe never superaccelerates. Further details of this intriguing corpuscular cosmology of the early universe will be reported elsewhere.

\section{Acknowledgments}

R.C. is partially supported by the INFN grant FLAG. This work has also been carried out in the framework of activities of the National Group of Mathematical Physics (GNFM, INdAM) of Italy. V.F. and A.G. are supported by the Natural Sciences and Engineering Research Council of Canada (Grant No. 2016-03803 to V.F.) and by Bishop's University.

\section{References}

[1] A.A. Starobinsky, "A New Type of Isotropic Cosmological Models Without Singularity," Phys. Lett. 91B (1980) 99.

[2] A.A. Starobinsky, "Spectrum of relict gravitational radiation and the early state of the universe," JETP Lett. 30 (1979) 682 [Pisma Zh. Eksp. Teor. Fiz. 30 (1979) 719].

[3] A.H. Guth, "The Inflationary Universe: A Possible Solution to the Horizon and Flatness Problems," Phys. Rev. D 23 (1981) 347.

[4] A.D. Linde, "A New Inflationary Universe Scenario: A Possible Solution of the Horizon, Flatness, Homogeneity, Isotropy and Primordial Monopole Problems," Phys. Lett. 108B (1982) 389.

[5] A. Albrecht and P.J. Steinhardt, "Cosmology for Grand Unified Theories with Radiatively Induced Symmetry Breaking," Phys. Rev. Lett. 48 (1982) 1220.

[6] H. Motohashi, A.A. Starobinsky and J. Yokoyama, "f(R) Gravity and its Cosmological Implications," Int. J. Mod. Phys. D 20 (2011) 1347 [arXiv:1101.0716 [astro-ph.CO]].

[7] T. P. Sotiriou and V. Faraoni, "f(R) Theories Of Gravity," Rev. Mod. Phys. 82 (2010) 451 [arXiv:0805.1726 [gr-qc]].

[8] A. De Felice and S. Tsujikawa, "f(R) theories," Living Rev. Rel. 13, 3 (2010) [arXiv:1002.4928 [gr-qc]].

[9] S. Nojiri and S. D. Odintsov, "Unified cosmic history in modified gravity: from F(R) theory to Lorentz non-invariant models," Phys. Rept. 505, 59 (2011) [arXiv:1011.0544 [gr-qc]].

[10] P.A.R. Ade et al. [Planck Collaboration], Astron. Astrophys. 594 (2016) A20 [arXiv:1502.02114 [astroph.CO]l.

[11] C. L. Bennett et al. [WMAP Collaboration], "Nine-Year Wilkinson Microwave Anisotropy Probe (WMAP) Observations: Final Maps and Results," Astrophys. J. Suppl. 208, 20 (2013) [arXiv:1212.5225 [astro-ph.CO]].

[12] G. Dvali and C. Gomez, JCAP 1401 (2014) 023 [arXiv:1312.4795 [hep-th]].

[13] G. Dvali and C. Gomez, "Black Hole's Quantum N-Portrait," Fortsch. Phys. 61 (2013) 742 [arXiv:1112.3359 [hep-th]].

[14] G. Dvali and C. Gomez, "Black Hole's 1/N Hair," Phys. Lett. B 719 (2013) 419 [arXiv:1203.6575 [hep-th]]. 
[15] G. Dvali and C. Gomez, "Black Holes as Critical Point of Quantum Phase Transition," Eur. Phys. J. C 74 (2014) 2752 [arXiv:1207.4059 [hep-th]].

[16] A. Giusti, "On the Corpuscular Theory of Gravity," Int. J. Geom. Meth. Mod. Phys. 16 (2019) 1930001.

[17] R. Casadio, A. Giugno and A. Giusti, "Matter and gravitons in the gravitational collapse," Phys. Lett. B 763 (2016) 337 [arXiv:1606.04744 [hep-th]].

[18] R. Casadio, A. Giugno, A. Giusti and M. Lenzi, "Quantum corpuscular corrections to the Newtonian potential," Phys. Rev. D 96 (2017) 044010 [arXiv:1702.05918 [gr-qc]].

[19] J.D. Bekenstein, "Black holes and entropy," Phys. Rev. D 7 (1973) 2333.

[20] R. Casadio, A. Giugno and A. Orlandi, "Thermal corpuscular black holes," Phys. Rev. D 91 (2015) 124069 [arXiv:1504.05356 [gr-qc]].

[21] R. Casadio, A. Giugno, O. Micu and A. Orlandi, "Thermal BEC black holes," Entropy 17 (2015) 6893 [arXiv:1511.01279 [gr-qc]].

[22] R. Casadio, F. Kuhnel and A. Orlandi, "Consistent Cosmic Microwave Background Spectra from Quantum Depletion," JCAP 1509 (2015) 002 [arXiv:1502.04703 [gr-qc]].

[23] M. Cadoni, R. Casadio, A. Giusti, W. Mück and M. Tuveri, "Effective Fluid Description of the Dark Universe," Phys. Lett. B 776 (2018) 242 [arXiv:1707.09945 [gr-qc]].

[24] M. Cadoni, R. Casadio, A. Giusti and M. Tuveri, "Emergence of a Dark Force in Corpuscular Gravity," Phys. Rev. D 97, no. 4, 044047 (2018) [arXiv:1801.10374 [gr-qc]].

[25] R. Casadio, A. Giugno and A. Giusti, "Corpuscular slow-roll inflation," Phys. Rev. D 97 (2018) no.2, 024041 [arXiv:1708.09736 [gr-qc]].

[26] A. Giugno and A. Giusti, "Domestic Corpuscular Inflaton," Int. J. Geom. Meth. Mod. Phys. to appear (2019) 1950108 [arXiv:1806.11168 [gr-qc]].

[27] G. Dvali and C. Gomez, "On Exclusion of Positive Cosmological Constant," Fortsch. Phys. 67, no. 1-2, 1800092 (2019) [arXiv:1806.10877 [hep-th]].

[28] J. D. Barrow and A. C. Ottewill, "The Stability of General Relativistic Cosmological Theory," J. Phys. A 16, 2757 (1983).

[29] M. Rinaldi, G. Cognola, L. Vanzo and S. Zerbini, "Inflation in scale-invariant theories of gravity," Phys. Rev. D 91 (2015) 123527 [arXiv:1410.0631 [gr-qc]].

[30] L. Alvarez-Gaume, A. Kehagias, C. Kounnas, D. Lüst and A. Riotto, "Aspects of Quadratic Gravity," Fortsch. Phys. 64 (2016) 176 [arXiv:1505.07657 [hep-th]].

[31] G. Cognola, E. Elizalde, S. Nojiri, S. D. Odintsov and S. Zerbini, JCAP 0502, 010 (2005) doi:10.1088/1475-7516/2005/02/010 [hep-th/0501096]. 